\title{
Local Energy Decay for the Wave Equation
}

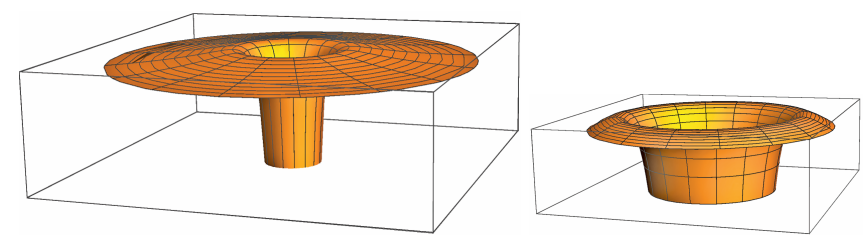

\section{Jason Metcalfe}

\section{W} e want to understand how waves spread out in spaces that look like Minkowski space-time as you go to infinity. This is the Lorentzian analog of being asymptotically Euclidean. To begin, we consider solutions to the Minkowski wave equation on $\mathbb{R} \times \mathbb{R}^{3}$ :

$$
\square u:=\frac{\partial^{2}}{\partial t^{2}} u-\Delta u=0,
$$

where $\Delta$ denotes the Laplacian

$$
\Delta u=\frac{\partial^{2} u}{\partial x_{1}^{2}}+\frac{\partial^{2} u}{\partial x_{2}^{2}}+\frac{\partial^{2} u}{\partial x_{3}^{2}} .
$$

Such solutions enjoy a conserved energy $E(t)=E(0)$,

$$
E(t)=\frac{1}{2} \int_{\mathbb{R}^{3}}\left(\partial_{t} u\right)^{2}+\left|\nabla_{x} u\right|^{2} d x .
$$

This energy is the same for all times; it does not decay.

On the other hand,

Imagine a stone thrown into the center of an infinite still ocean if the domain of integration is restricted to a compact set, say $B_{R}=\{|x| \leq R\}$, we expect the energy $E_{B_{R}}(t)$ within that set eventually to decrease in time. Imagine a stone thrown into the center of an infinite still ocean. After an initial splash, the waves move away, the center calms, and the energy within that set decreases. For some absolute constant $C$, if the total energy $E(0)$ is finite, say 1 , then $E_{B_{R}}(t)$ has a finite integral

Jason Metcalfe is associate professor of mathematics at the University of North Carolina. His email address is metcalfe@ emai 1 . unc.edu.

This research is supported by the NSF CAREER award DMS1054289.

For permission to reprint this article, please contact:

reprint-permission@ams.org.

DOI: http://dx.doi.org/10.1090/noti1437 with respect to $t$; indeed, its integral is bounded by $C R$. Such estimates originated in work of Cathleen Morawetz.

In joint works with Christopher Sogge and with Daniel Tataru, we proved that such estimates continue to hold for small perturbations of Minkowski space-time that decay as $|x| \rightarrow \infty$. Once such estimates are known, several other common measures of dispersion have been shown to follow in such spaces, including the so-called Strichartz estimates and pointwise decay estimates.

We now turn our attention to wave operators on such asymptotically flat backgrounds but where the size of the perturbation may not be universally small. Moreover, lower-order perturbations such as potentials are also allowed. Motivated by, e.g., Maxwell's equations, these lower-order perturbations are permitted to be complex valued.

We will describe two immediate obstructions to local energy decay. Recent work with Jacob Sterbenz and Tataru demonstrates that these are the only relevant obstructions. The first obstructions are certain possible eigenfunctions and resonances of the elliptic portion of the operator. The second obstruction is trapping, when a geodesic, and hence energy from a disturbance, can remain in a compact set for all time.

One place where trapping occurs is in black hole spacetimes from general relativity. In the simplest example, Schwarzschild space, as depicted in Figure 1, photons can orbit the black hole along the so-called photon sphere. Under magnification as in Figure 1(B), this appears as a ridge about the black hole. Recent work shows that because the trapped geodesics are unstable-nearby

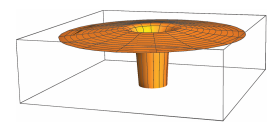

(A) Schwarzschild

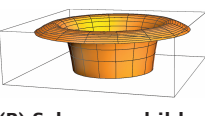

(B) Schwarzschild zoomed around the photon sphere
Figure 1. Photons orbit a black hole in Schwarzschild space along what appears as a ridge under magnification. 
geodesics go off to infinity or into the black holelocal energy decay can be recovered with a small loss. Stable trapping, however, eliminates most local energy decay. Interesting examples of in-between scenarios have recently been discovered, and a more general theory remains to be examined.

\section{Photo Credit}

Photo of Jason Metcalfe, courtesy of Anita Hepditch.

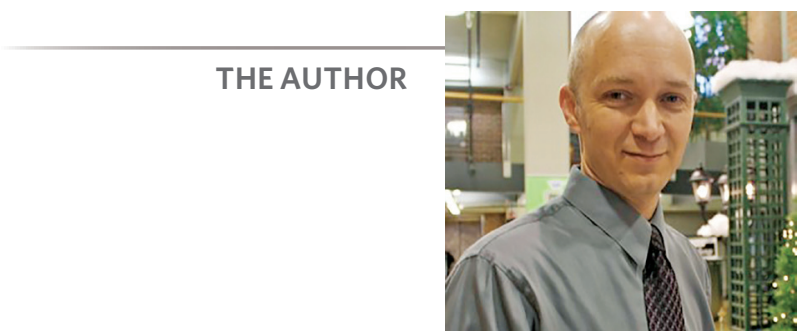

Jason Metcalfe

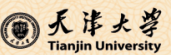

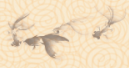

Tenured/Tenure-Track/Postdoctoral Positions at the Center for Applied Mathematics

Tianjin University, China

Dozens of positions at all levels are available at the recently founded Center for Applied Mathematics, Tianjin University, China. We welcome applicants with backgrounds in pure mathematics, applied mathematics, statistics, computer science, bioinformatics, and other related fields. We also welcome applicants who are interested in practical projects with industries. Despite its name attached with an accent of applied mathematics, we also aim to create a strong presence of pure mathematics. Chinese citizenship is not required.

Light or no teaching duties, adequate facilities, spacious office environment and strong research support. We are prepared to make quick and competitive offers to self-motivated hard workers, and to potential stars, rising stars, as well as shining

The Center for Applied Mathematics, also known as the Tianjin Center for Applied Mathematics (TCAM), located by a lake in the central campus in a building protected as historical architecture, is jointly sponsored by the Tianjin municipal government and the university. The initiative to establish this center was taken by Professor S. S. Chern. Professor Molin Ge is the Honorary Director, Professor Zhiming Ma is the Director of the Advisory Board. Professor William Y.C. Chen serves as the Director.

TCAM plans to fill in fifty or more permanent faculty positions in the next few years. In addition, there are a number of temporary and visiting positions. We look forward to receiving your application or inquiry at any time. There are no deadlines.

For more information, please visit http://www.cam.tju.edu.cn// or contact Ms. Debbie Renyuan Zhang at zhangry@tju.edu.cn, telephone: 86-22-2740-5389.

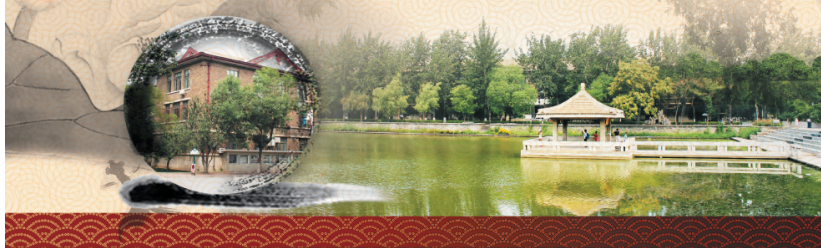

